\title{
Valoración del Morfotipo Raquídeo en el Plano Sagital en Ciclistas de Categoría Máster 40
}

\author{
Spinal Posture of Thoracic and Lumbar Spine in Master 40 Cyclists \\ *José María Muyor; **Fernando Alacid \& ${ }^{* * * *}$ Pedro Ángel López-Miñarro
}

MUYOR, J. M.; ALACID, F.; LÓPEZ-MIÑARRO, P. A. Valoración del morfotipo raquídeo en el plano sagital en ciclistas de categoría máster 40. Int. J. Morphol., 29(3):727-732, 2011.

RESUMEN: El objetivo principal del estudio fue valorar la disposición sagital del raquis torácico y lumbar en bipedestación y sobre la bicicleta, en ciclistas de la categoría máster 40. Un total de 50 ciclistas máster 40 (media de edad: 44,02 $\pm 2,51$ años) fueron evaluados mediante el sistema Spinal Mouse en bipedestación y sobre la bicicleta en tres agarres del manillar: transversal, de manetas y bajo. En bipedestación, los valores angulares medios para el raquis torácico y lumbar fueron de $49,42 \pm 9,00^{\circ}$ y $-22,74 \pm 9,38^{\circ}$, respectivamente. Un elevado porcentaje de los ciclistas $(68 \%)$ presentaron una hipercifosis torácica, mientras que la mayoría tenían valores normales en la lordosis lumbar. Sobre la bicicleta, los ciclistas mostraron una reducción significativa de la cifosis torácica con respecto a la bipedestación, mientras que el raquis lumbar se disponía en una postura de inversión. En conclusión, la frecuente hipercifosis torácica en bipedestación de los ciclistas de la categoría máster 40 no está relacionada directamente con la postura adoptada sobre la bicicleta.

PALABRAS CLAVE: Ciclismo; Cifosis; Lordosis; Columna vertebral; Postura.

\section{INTRODUCCIÓN}

El raquis presenta una serie de curvaturas en el plano sagital de naturaleza fisiológica (Miralles, 1998), que están condicionadas por la morfología de los cuerpos vertebrales, la funcionalidad de los discos intervertebrales, las estructuras ligamentosas y la integridad anatomo-fisiológica de la musculatura (Sañudo et al., 1985). La práctica deportiva de alta intensidad, realizada de manera sistemática se ha asociado con adaptaciones en la morfología sagital del raquis (Wojtys et al., 2000).

En deportes donde predominan posturas mantenidas en flexión del tronco, tales como esquí de fondo, remo y lucha se ha encontrado una mayor cifosis torácica en bipedestación en comparación con un grupo control de sujetos sedentarios (Alricsson \& Werner, 2006; Rajabi et al., 2008; Stutchfield \& Coleman, 2006).

El ciclismo es un deporte muy practicado, a pesar de ser una disciplina donde influyen diversos factores en su realización, tales como altas exigencias físicas, climatología, interacción con vehículos de motor (Winters et al., 2010) y la adopción de una postura considerada como antinatural. En este deporte, la posición predominante es la sedentación so- bre la bicicleta, con flexión del tronco para apoyar las manos en el manillar. Esta postura podría generar adaptaciones en la morfología raquídea. Salai et al. (1999) observaron que más del $50 \%$ de los hombres y mujeres ciclistas presentaban algias lumbares, posiblemente asociadas a la posición que adopta el ciclista sobre la bicicleta. La sedentación prologada, junto a cargas intensas de entrenamiento se ha asociado a una deformación viscoelástica de los tejidos espinales (Solomonow et al., 2003), mayor estrés vertebral (Beach et al., 2005) y presión intradiscal (Wilke et al., 1999).

Hasta la actualidad, son pocos los estudios que han valorado el morfotipo raquídeo en ciclistas. En este sentido, Rajabi et al. (2000) encontraron una mayor y significativa cifosis torácica en bipedestación en ciclistas, en comparación con un grupo de sujetos sedentarios. Sin embargo, no analizaron la disposición del raquis lumbar ni la posición específica en la bicicleta. Usabiaga et al. (1997) estudiaron la posición del raquis lumbar de ciclistas profesionales al sentarse en la bicicleta, encontrando un cambio de lordosis en bipedestación, a una inversión en la bicicleta. Sin embargo, no analizaron la morfología del raquis torácico ni la influencia del tipo de agarre en la postura adoptada.

*Área de Expresión Corporal. Facultad de Ciencias de la Educación. Universidad de Almería. España.

**Eepartamento de Actividad Física y Deporte. Facultad de Ciencias del Deporte. Universidad de Murcia. España.

*** Departamento de Expresión Plástica, Musical y Dinámica. Facultad de Educación. Universidad de Murcia. España. 
Puesto que el ciclista mantiene durante varias horas al día, tanto en los entrenamientos como en las competiciones, una postura de sedentación sobre su bicicleta con una ligera flexión del tronco que podría condicionar su morfología raquídea, el objetivo del presente trabajo fue evaluar la disposición angular del raquis torácico y lumbar en bipedestación y sobre la bicicleta, en ciclistas de la categoría máster 40.

\section{MATERIAL Y MÉTODO}

Un total de 50 ciclistas de categoría máster 40, entre 40-49 años de edad (media de edad: 44,02 $\pm 2,51$ años), participaron voluntariamente en este estudio. Los datos descriptivos de la muestra se presentan en la Tabla I. Los criterios de inclusión de los ciclistas para participar en este estudio fueron: estar federados por la Real Federación Española de Ciclismo en la categoría máster 40 , tener un volumen de entrenamiento de 2 a 4 horas al día, con una frecuencia de 2 a 6 días por semana, y un historial de entrenamiento en ciclismo de más de 8 años de práctica ininterrumpida. Los criterios de exclusión fueron: 1) haber padecido algún episodio de dolor raquídeo en los 3 meses previos a la participación en el estudio; 2) haber sido operado de la columna vertebral; o 3 ) tener alguna patología raquídea diagnosticada. Todos los sujetos fueron instruidos a no participar en actividades físicas o entrenamientos intensos 24 horas antes a las mediciones.

Tabla I. Datos descriptivos de la muestra.

$\begin{array}{lr}\text { Edad (años) } & 44,02 \pm 2,51 \\ \text { Talla (m) } & 1,75 \pm 0,05 \\ \text { Masa }(\mathrm{kg}) & 76,86 \pm 9,79 \\ \mathrm{IMC}\left(\mathrm{kg} \cdot \mathrm{m}^{-2}\right) & 25,07 \pm 2,56 \\ \text { Entrenamiento (años) } & 12,78 \pm 9,44 \\ \text { Entrenamiento (días/semana) } & 3,12 \pm 1,23 \\ \text { Entrenamiento (horas/día) } & 2,83 \pm 0,84\end{array}$

Procedimiento. El estudio fue aprobado por la Comisión de Bioética de la Universidad de Almería. Previamente a las mediciones, todos los sujetos fueron informados sobre el procedimiento y firmaron, voluntariamente, un consentimiento informado.

Para la valoración de la disposición angular de la curva torácica y lumbar en bipedestación y sobre la bicicleta en los diferentes agarres del manillar (transversal, de manetas y bajo), se utilizó el sistema Spinal Mouse ${ }^{\circledR}$ (Idiag, Switzerland). Cada sujeto fue valorado en ropa interior, descalzo y por el mismo examinador en una misma sesión. La vestimenta utilizada en la valoración sobre la bicicleta fue un culote sin tirantas y las propias zapatillas automáticas de los ciclistas. La temperatura fue estandarizada a $24^{\circ} \mathrm{C}$. Las mediciones se realizaron en un orden aleatorio y hubo $5 \mathrm{mi}-$ nutos de descanso entre cada una de ellas.

Previamente a las mediciones, el investigador principal identificó mediante palpación y marcó, con un lápiz dérmico, el proceso espinoso de la séptima vértebra cervical (C7), así como la tercera vértebra sacra (S3). Para medir las curvas raquídeas, una vez que el sujeto se colocaba en la posición a medir, se guiaba el Spinal Mouse ${ }^{\circledR}$ a lo largo de los procesos espinosos del raquis, desde C7 hasta S3. El sistema digitalizaba el contorno de la piel sobre el raquis en el plano sagital, aportando información sobre la angulación global de las curvas raquídeas. Con respecto a la curva lumbar, los valores negativos indicaron angulaciones de concavidad posterior (lordosis), mientras que los valores positivos correspondieron a una curvatura de convexidad posterior (inversión lumbar).

\section{Posturas}

Bipedestación: Los sujetos se situaban de pie, con los hombros relajados, mirada al frente, los brazos a lo largo del tronco y con una apertura de los pies igual a la anchura de sus caderas. Para categorizar los valores angulares de la curva torácica en bipedestación en base a unas referencias de normalidad, se utilizó la propuesta de Mejia et al. (1996): cifosis torácica normal (entre $20^{\circ}$ y $45^{\circ}$ ), hipocifosis torácica (< $20^{\circ}$ ), e hipercifosis torácica $\left(>45^{\circ}\right)$. Respecto a la curva lumbar, valores entre $20^{\circ}$ y $40^{\circ}$ fueron considerados como lordosis normal, mientras que valores inferiores a $20^{\circ}$ se consideraron como hipolordosis y valores superiores a $40^{\circ}$ como hiperlordosis (Tüzün et al., 1999).

Sedentación sobre la bicicleta: Cada ciclista utilizó su propia bicicleta. Los sujetos debían sentarse en el sillín y pedalear durante 5 minutos, a una cadencia de 90 pedaladas por minuto (marcadas con un cadenciómetro). Se midió la morfología de las curvas torácica y lumbar en tres posiciones, en función del tipo de agarre en el manillar: agarre transversal, agarre de manetas y agarre bajo (Fig. 1). Las mediciones se realizaron en un orden aleatorio. Entre cada posición hubo un descanso de 30 segundos.

Análisis estadístico. Los valores medios y desviaciones típicas fueron calculados para todas las variables. La hipótesis de normalidad fue analizada mediante el test Kolmogorov-Smirnov. Un análisis de varianza (ANOVA) fue usado para comparar la disposición sagital del raquis en las diferentes posiciones evaluadas. Si se obtenía un p-valor significativo para el efecto principal del ANOVA, se proce- 


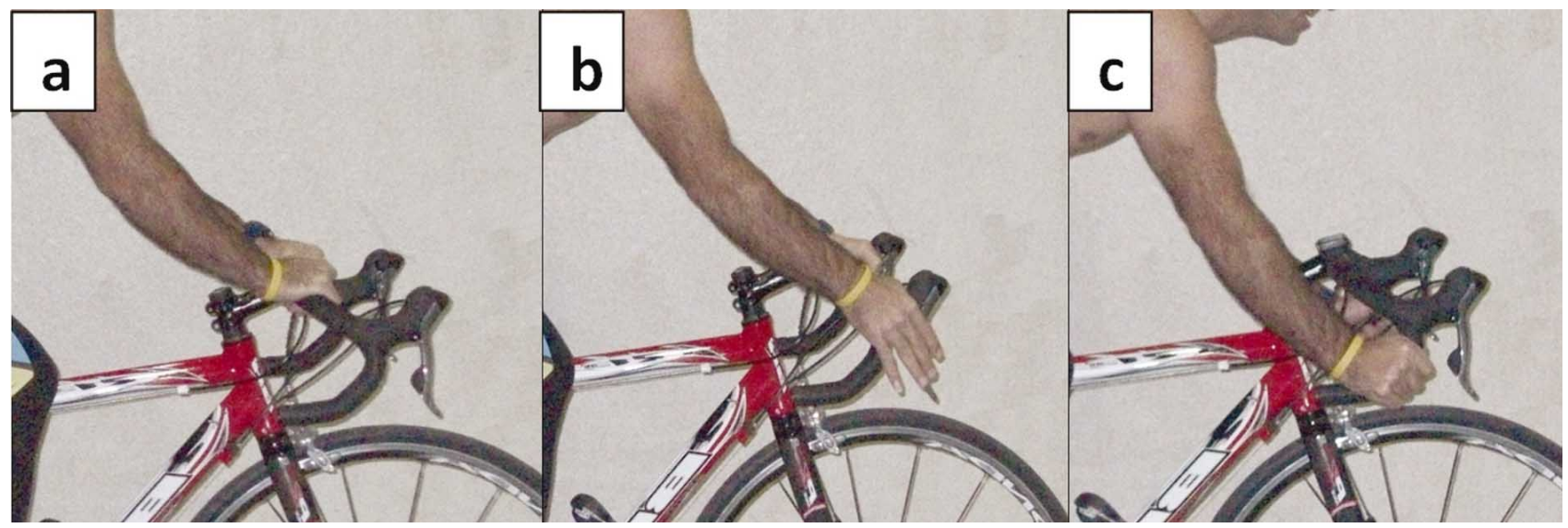

Fig. 1. Posiciones en el manillar: agarre transversal (a), agarre de manetas (b), y agarre bajo (c).

dió a realizar una comparación por pares (post hoc) usando la corrección de Bonferroni para comparaciones múltiples, ajustando el criterio de significación a un valor de 0,0125 $(0,05 / 4)$. Los datos fueron analizados usando el software SPSS, v. 15.0 y el nivel de significación, a priori, fue de $\mathrm{p}<$ 0,05 .

\section{RESULTADOS}

Los valores medios ( \pm desviación típica) de la curva torácica y lumbar en las diferentes posiciones analizadas, se presenta en la Tabla II. El ANOVA de medidas repetidas reveló diferencias significativas para el efecto principal en el raquis torácico y lumbar ( $\mathrm{p}<0,05)$. El análisis post hoc con ajuste de Bonferroni mostró una significativa y mayor cifosis torácica en bipedestación que la adoptada sobre la bicicleta en los tres agarres del manillar analizados $(\mathrm{p}<$ $0,0125)$. La flexión del raquis lumbar fue significativamente mayor a medida que el agarre en el manillar era más bajo con respecto a la altura del sillín (Tabla III). Los porcentajes de casos en función de las referencias de normalidad para el raquis torácico y lumbar en la postura de bipedestación se presentan en la Figura 2.

Tabla III. Comparación por pares entre las diferentes posiciones para el raquis torácico, lumbar e inclinación pélvica.

\begin{tabular}{lllll} 
& & Agarre transversal & Agarre de manetas & Agarre bajo \\
\hline \multirow{2}{*}{ Raquis torácico } & Bipedestación & $*$ & $*$ & NS \\
& Agarre transversal & - & - & NS \\
& Agarre de manetas & & $*$ & NS \\
& Bipedestación & $*$ & $*$ & $*$ \\
Raquis lumbar & Agarre transversal & - & - & $*$
\end{tabular}

Tabla II. Valores medios del raquis torácico, lumbar e inclinación pélvica en las cuatro posturas evaluadas.

$\begin{array}{lll}\text { Bipedestación } & \text { Raquis torácico } & 49,42 \pm 9,00^{\circ} \\ & \text { Raquis lumbar } & -22,74 \pm 9,38^{\circ} \\ \text { Agarre transversal } & \text { Raquis torácico } & 43,84 \pm 8,35^{\circ} \\ & \text { Raquis lumbar } & 20,70 \pm 10,01^{\circ} \\ \text { Agarre de manetas } & \text { Raquis torácico } & 41,98 \pm 8,43^{\circ} \\ & \text { Raquis lumbar } & 22,38 \pm 10,47^{\circ} \\ \text { Agarre bajo } & \text { Raquis torácico } & 42,78 \pm 9,32^{\circ} \\ & \text { Raquis lumbar } & 23,96 \pm 9,89^{\circ}\end{array}$

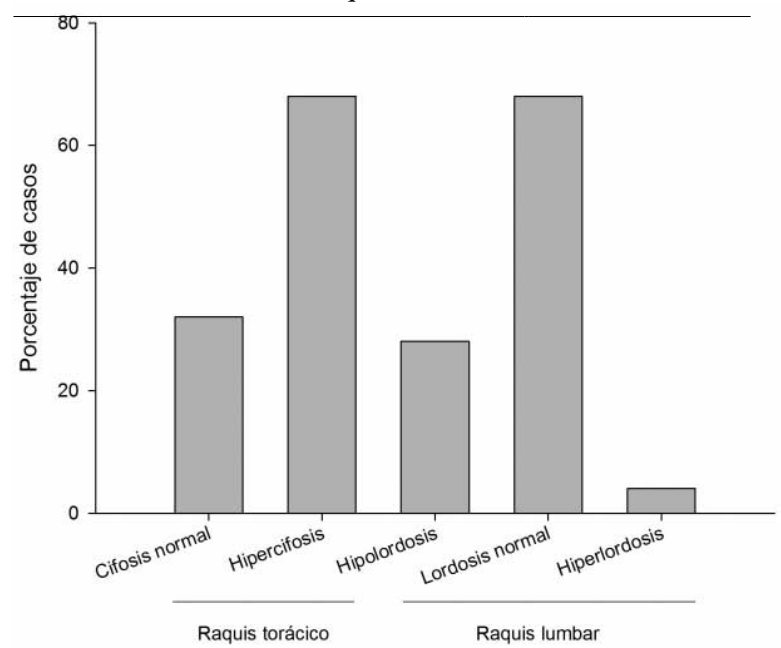

Fig. 2. Porcentaje de sujetos en función de cada categoría para el raquis torácico y lumbar en bipedestación. 


\section{DISCUSIÓN}

Uno de los principales hallazgos del presente estudio fue la alta frecuencia de ciclistas con una hipercifosis torácica en bipedestación $(68 \%)$, mientras que respecto a la curva lumbar, la mayoría de estos deportistas tenían valores considerados normales. En otros deportes donde predominan gestos técnicos específicos en flexión mantenida o cíclica del tronco, como en lucha, piragüismo, voleibol y esquí, se ha observado una tendencia al aumento de la cifosis torácica en bipedestación (Rajabi et al., 2008; LópezMiñarro \& Alacid, 2010; López-Miñarro et al., 2010; Grabara \& Hadzik, 2009, Alricsson \& Werner). Sin embargo, en estos trabajos no se analizó la posición adoptada en los gestos deportivos específicos. Rajabi et al. (2000) observaron, en ciclistas de élite, una cifosis torácica significativamente mayor con respecto a un grupo control de sujetos que no practicaban deporte, justificando estas diferencias por el tiempo que pasan los ciclistas en flexión del tronco sobre la bicicleta, generándose adaptaciones en la morfología raquídea. Alricsson \& Werner observaron un incremento de la cifosis torácica en esquiadores adolescentes tras 5 años de entrenamiento intenso. En el presente estudio, los ciclistas tenían una experiencia media de 12 años de entrenamiento en ciclismo, por lo que podría pensarse que la hipercifosis torácica encontrada en estos ciclistas se debe a una adaptación del raquis sobre la postura mantenida durante tantos años de entrenamiento sobre la bicicleta.

En el presente estudio, al analizar las curvas sagitales del raquis sobre la bicicleta, en los tres agarres evaluados, el raquis torácico manifestó una significativa menor cifosis que en bipedestación (diferencias de medias: $5,58^{\circ}, 7,44^{\circ}$ y $6,64^{\circ}$ en el agarre transversal, de manetas y bajo, respectivamente). El ciclismo ha sido clasificado como un deporte vertebralmente negativo en potencia (Balius et al., 1987) debido a que su práctica comporta una inevitable posición en hipercifosis torácica, que puede desencadenar modificaciones raquídeas cuando su práctica es intensa y continuada (Balius, 1970; 1983). Sin embargo, los datos obtenidos de este estudio no sustentan dichas afirmaciones. La menor cifosis torácica en la bicicleta podría estar relacionada con la aducción y retropulsión de la cintura escapular, así como con la extensión intervertebral torácica generada al apoyar las manos sobre el manillar. Por ello, el elevado porcentaje de casos con hipercifosis torácica encontrados en bipedestación estarían condicionados por otros factores como la edad, hábitos posturales, estilo de vida, o un inadecuado esquema corporal, más que a la adaptación de las estructuras raquídeas debido a la postura adoptada sobre la bicicleta. No obstante, es preciso valorar si la po- sición del ciclista mantenida durante tantas horas cada día, puede generar, por la fatiga asociada, modificaciones posturales tras los entrenamientos, explicando en parte su morfología raquídea en bipedestación.

Una cifosis torácica aumentada en deportistas cuyo entrenamiento se caracteriza por un predominio de posturas de flexión del tronco, se explica por la pérdida en la altura de los discos intervertebrales, los cuales tenderían a la reducción de la longitud anterior de la columna vertebral (Rajabi et al., 2008; Wojtys et al.). Este incremento de la flexión intervertebral torácica provoca un aumento de la presión intradiscal y estrés raquídeo, así como una mayor posibilidad de lesiones raquídeas (Briggs et al., 2007).

$\mathrm{Al}$ analizar la morfología del raquis lumbar sobre la bicicleta, se encontró una postura de inversión lumbar en los tres agarres analizados, siendo mayor a medida que el agarre sobre el manillar era más distal y bajo con respecto al apoyo sobre el sillín de la bicicleta. Usabiaga et al. tras valorar mediante radiografías a tres ciclistas profesionales sobre sus bicicletas, evidenciaron en todos ellos un raquis lumbar en inversión. La posición del raquis en los ciclistas se ha relacionado con la búsqueda de una mayor aerodinámica. Sobre la bicicleta, el raquis lumbar se flexiona para poder apoyar las manos sobre el manillar que, normalmente, se sitúa más bajo que la altura del sillín. Un alto volumen de entrenamiento con el raquis lumbar en inversión podría generar adaptaciones raquídeas específicas que deriven en una disminución de la lordosis lumbar en bipedestación. En el presente estudio, el 28\% de los ciclistas presentaban hipolordosis lumbar en bipedestación. Ogurkowska (2007) observó, en remeros con una experiencia de práctica deportiva de 12 años de media (mínimo: 8 años y máximo: 20 años), cambios en las alturas de los cuerpos vertebrales y discos intervertebrales del raquis lumbar que modificaban su morfología raquídea. Dichas modificaciones raquídeas podrían generar alteraciones de la carga y movilidad intersegmentaria, posiblemente favoreciendo una degeneración discal (Keorochana et al., 2011). Mantener el raquis lumbar en una postura de inversión durante largos periodos de tiempo, podría alterar la distribución de carga raquídea (Keller et al., 2005), aumentar la presión intradiscal (Wilke et al., 1999), deformar los tejidos espinales visco-elásticos (Caldwell \& Peters, 2009; Solomonov et al.), así como producir un aumento del dolor raquídeo en ciclistas (Marsden \& Schwellnus, 2010).

En conclusión, los ciclistas de categoría máster 40 presentan un elevado porcentaje de casos con hipercifosis 
torácica y lordosis lumbar normal en bipedestación, aunque un moderado porcentaje muestran una hipolordosis lumbar. Sobre la bicicleta, en los diferentes agarres del manillar, los ciclistas máster 40 colocan el raquis torácico en una posición más alineada que en bipedestación, mientras que el raquis lumbar se dispone en inversión, acentuándose la misma, cuanto más distal y bajo es el agarre en el manillar con respecto al sillín de la bicicleta.

MUYOR, J. M.; ALACID, F.; LÓPEZ-MIÑARRO, P. A. Spinal posture of thoracic and lumbar spine in master 40 cyclists. Int. J. Morphol., 29(3):727-732, 2011.

SUMMARY: The aim of this study was to determine the sagittal spinal morphology of thoracic and lumbar spine in relaxed standing and sitting on the bycicle in master 40 cyclists. A total of 50 master 40 male cyclists (mean age: $44.02 \pm 2.51$ years) were evaluated. The Spinal Mouse system was used to measure the sagittal thoracic and lumbar curve in standing and sitting on the bicycle at three different handlebar-hand positions (high, medium, and low). The values for thoracic and lumbar curvatures in standing were 49.42 $\pm 9.00^{\circ}$ and $-22.74 \pm 9.38^{\circ}$, respectively. A high frecuency of thoracic hyperkyphosis in standing was observed (68\%). When sitting on the bicycle the thoracic curve showed lower angles in the three handlebar.hand positions that in standing. The lumbar curve adopted a kyphotic posture. The standing thoracic hyperkyphosis in master 40 cyclists may be related to other factors than the posture adopted on the bicycle.

KEY WORDS: Cycling; Kyphosis; Lordosis; Spine; Posture.

\section{REFERENCIAS BIBLIOGRÁFICAS}

Alricsson, M. \& Werner, S. Young élite cross-country skiers and low back pain. A 5-year study. Phys. Ther. Sport., 7(4):181-4, 2006.

Balius, R. Alteraciones que predisponen a la patología. Apunts Med. de'l Esport, 7(26):99-115, 1970.

Balius, R. Acción de la sobrecarga deportiva sobre el aparato locomotor del niño y del adolescente. Apunts, 20(78):85-96, 1983.

Balius, R.; Balius, R. \& Balius, X. Columna vertebral y deporte. Apunts, 24:223-9, 1987.

Beach, T.; Parkinson, R.; Stothart, P. \& Callaghan, J. Effects of prolonged sitting on the passive flexion stiffness of the in vivo lumbar spine. Spine J., 5(2):145$54,2005$.

Briggs, A.; Van Dieën, J.; Wrigley, T.; Greig, A.; Phillips, B.; Lo, S. \& Bennell, K. Thoracic kyphosis affects spinal loads and trunk muscle force. Phys. Ther., 87(5):595-607, 2007.

Caldwell, B. \& Peters, D. Seasonal variation in physiological fitness of a semiprofessional soccer team. J. Strength Cond. Res., 23(5):1370-7, 2009.

Grabara, M. \& Hadzik, A. Postural variables in girls practicing volleyball. Biomed. Hum. Kinet., 1(1):6771,2009 .
Keller, T. S.; Colloca, C. J.; Harrison, D. E.; Harrison, D. D. \& Janik, T. J. Influence of spine morphology on intervertebral disc loads and stresses in asymptomatic adults: implications for the ideal spine. Spine J., 5(3):297-300, 2005.

Keorochana, G.; Taghavi, C. E.; Lee, K. B.; Yoo, J. H.; Liao, J.; Fei, Z. \& Wang, J. C. Effect of sagittal alignment on kinematic changes and degree of disc degeneration in the lumbar spine: An analysis using positional MRI. Spine, 36(11):893-8, 2011.

López-Miñarro, P. A. \& Alacid, F. Influence of hamstring muscle extensibility on spinal curvatures in young athletes. Sci. Sports, 25(4):88-93, 2010.

López-Miñarro, P. A.; Alacid, F. \& Rodríguez-García, P. L. Comparison of sagittal spinal curvatures and hamstring muscle extensibility among young elite paddlers and non-athletes. Int. Sport Med. J., 11(2):301-12, 2010.

Marsden, M. \& Schwellnus, M. Lower back pain in cyclists: A review of epidemiology pathomechanics and risk factors. Int. Sport Med. J., 11(1):216-25, 2010 .

Mejia, E. A.; Hennrikus, W. L.; Schwend, R. M. \& Emans, J. B. A prospective evaluation of idiopathic left thoracic scoliosis with MRI. J. Pediatr. Orthop., 16(3):354-8, 1996. 
Miralles, R. Biomecánica clínica del aparato locomotor. Barcelona, Masson, 1998.

Ogurkowska, M. B. Pathological change of intervertebral disc of the lumbosacral spine of competitive rowers. Biol. Sport, 24:375-388, 2007.

Rajabi, R.; Doherty, P.; Goodarzi, M. \& Hemayattalab, R. Comparison of thoracic kyphosis in two groups of élite Greco-Roman and free style wrestlers and a group of non-athletic subjects. Br. J. Sports Med., 42(3):229-32, 2008.

Rajabi, R.; Freemont, A. \& Doherty, P. The investigation of cycling position on thoracic spine. A novel method of measuring thoracic kyphosis in the standing position. Arch. Physiol. Biochem., 1:142, 2000.

Salai, M.; Brosh, T.; Blankstein, A.; Oran, A. \& Chechik, A. Effect of changing the saddle angle on the incidence of low back pain in recreational bicyclists. Br. J. Sports Med., 33(6):398-400, 1999.

Sañudo, J.; Rodríguez, A. \& Domenech, J. Anatomía y embriología de la columna vertebral. En: Viladot, R. \& Cohi, O. Ortesis y prótesis del aparato locomotor. Barcelona, Masson, 1992. pp.13-27.

Solomonow, M.; Zhou, B.; Baratta, R. V. \& Burger, E. Biomechanics and electromyography of a cumulative lumbar disorder: response to static flexion. Clin. Biomech., 18(19): 883-9, 2003.

Stutchfield, B. \& Coleman, S. The relationships between hamstring flexibility, lumbar flexion, and low back pain in rowers. Eur. J. Sports Sci., 6(4):255-60, 2006.

Tüzün, C.; Yorulmaz, I.; Cindas , A. \& Vatan, S. Low back pain and posture. Clin. Rheumatol., 18(4):308-12, 1999.

Usabiaga, J.; Crespo, R.; Iza, I.; Aramendi, J.; Terrados, N. \& Poza, J. Adaptation of the lumbar spine to different positions in bicycle racing. Spine, 22(17):1965-9, 1997.

Wilke, H.; Neef, P.; Caimi, M.; Hoogland, T. \& Claes, L. New in vivo measurements of pressures in the intervertebral disc in daily life. Spine, 24(8):755-62, 1999.

Winters, M.; Davidson, G.; Kao, D. \& Teschke, K. Motivators and deterrents of bicycling: comparing influences on decisions to ride. Transportation, 38(1):153-68, 2010.
Wojtys, E.; Ashton-Miller, J.; Huston, L. \& Moga, P. The association between athletic training time and sagittal curvature of the immature spine. Am. J. Sports Med., 28(4):490-8, 2000.

Dirección para correspondencia:

José María Muyor

Universidad de Almería

Área Didáctica de la Expresión Corporal

Edificio Central, Despacho 0.47

Ctra. Sacramento S/N, 04120.

La Cañada de San Urbano

Almería

ESPAÑA

Tel: 34620302928

Email: josemuyor@ual.es

Recibido : 23-01-2011

Aceptado: 06-04-2011 